\title{
Positive conditioned suppression as a function of the percentage of CS-US pairings
}

\author{
MARC LEVINSON and DONALD MELTZER \\ Southern Mlinois University, Carbondale, Illinois 62901
}

\begin{abstract}
Two groups of 12 rats were trained to barpress on a variable-interval 2-min schedule of sucrose solution reinforcement. While responding on this schedule of reinforcement, the subjects were exposed to a classical conditioning contingency, in which an 8-sec light CS terminated with the delivery of a food pellet US on either $100 \%$ or $50 \%$ of the CS presentations. Comparisons of the response rate during the CS to the response rate prior to the CS revealed that suppression of CS rate was directly related to the probability of CS terminating with US.
\end{abstract}

Estes and Skinner (1941) reported that the rate of a positively reinforced operant response was suppressed during a conditioned stimulus (CS) which previously had been paired with a negative unconditioned stimulus (US), such as electric shock. This conditioned suppression presumably occurred because the CS elicited a conditioned emotional response which was incompatible with the operant response. Since this first description, the negative conditioned suppression procedure has been used frequently to investigate the parameters which affect the interactions between operant and respondent conditioning.

A less frequently studied variation of the Estes and Skinner (1941) procedure consists of following the CS with a positive US, such as food, rather than a negative US, such as shock. Meltzer and Brahlek (1970) found that the rate of a positively reinforced operant response increased during a long-duration CS which preceded a positive US (condition enhancement), and decreased during a short-duration CS which preceded a positive US (positive conditioned suppression). Azrin and Hake (1969) suggested that a common emotional arousal may mediate both negative and positive conditioned suppression, and called for additional research investigating variables common to both procedures.

One variable common to both procedures is the percentage of CS-US pairings. A number of studies have reported that the rate of acquisition of negative conditioned suppression is directly related to the probability that the CS will terminate with electric shock (Brimer \& Dockrill, 1966; Geller, 1964). However, no significant differences in asymptotic negative conditioned suppression could be found between groups receiving different percentages of CS-US pairings. All groups eventually reached almost complete response suppression, even when as few as $25 \%$ of the CSs terminated with shock

Reprints may be obtained from Donald Meltzer, Department of Psychology, Southern Illinois University, Carbondale, Illinois 62901 .
(Hilton, 1969; Wagner, Seigel, \& Fein, 1967; Willis, 1969). The only study which reported a direct relationship between the percentage of CS-US pairings and negative conditioned suppression varied the probability of shock within subjects (Willis \& Lundin, 1966). It seems likely that a type of "floor effect" may be responsible for the failure to find differences between groups receiving different percentages of CS-US pairings. Once response rate during the CS approaches zero, higher probabilities of shock could not increase response suppression.

One previous experiment has investigated the effects of varying the probability of a positive US on positively reinforced operant response rate. Meltzer and Hamm (1974) found that asymptotic conditioned enhancement increased as the percentage of pairings of a long (2-min) light CS and a positive US increased from $33 \%$ to $66 \%$ to $100 \%$. The duration of the CS was then reduced to $8 \mathrm{sec}$, with the percentage of CS-US pairings remaining at the previous values for each group. However, the expected positive conditioned suppression did not develop during the short-duration CS. The 100\% group showed neither enhancement nor suppression, while the rates of the $33 \%$ and $66 \%$ groups were considerably enhanced during the CS. Meltzer and Hamm interpreted these results by suggesting that a CS which had been paired with a positive US always elicits a positive emotional response which facilitates positively reinforced operant responses. A second response, such as a postural adjustment or sign tracking (Hearst \& Jenkins, 1974), which interferes with the operant response may also be elicited by the CS. The strength of the second interfering response presumably increases with the percentage of CS-US pairings, and decreases with the duration of the CS. Meltzer and Hamm predicted that low probabilities of a positive US following a short CS would produce conditioned enhancement by reducing the sign-tracking tendencies and thus leaving the facilitating emotional response relatively unopposed.

The Meltzer and Hamm experiment confounded the effects of percentage of pairings of a short CS and a 
positive US with a shift in the duration of the CS. Accordingly, the present experiment sought to determine the effects of manipulating the percentage of CS-US pairings with no confounding previous experience.

\section{METHOD}

\section{Subjects}

The subjects were 24 experimentally naive male Long-Evans rats, approximately 120 days old at the beginning of the experiment. All rats were maintained at approximately $80 \%$ of their free-feeding weight. Water was continuously available in the subjects' individual home cages.

\section{Apparatus}

Rats were tested individually in standard operant conditioning chambers measuring $22.2 \times 19.1 \times 19.1 \mathrm{~cm}$. The front wall of each chamber had the following configuration: a response lever $3.2 \mathrm{~cm}$ to the right of center and $6.4 \mathrm{~cm}$ above the grid floor, an opening for access to a liquid dipper $4.4 \mathrm{~cm}$ from the left wall and $2.0 \mathrm{~cm}$ above the floor, a food pellet cup located $3.0 \mathrm{~cm}$ from the right wall and $3.0 \mathrm{~cm}$ above the floor, and a pilot light located $14.0 \mathrm{~cm}$ above the liquid-dipper opening. Each chamber was placed in a ventilated sound-attenuating enclosure. White noise was always present in the experimental room to mask extraneous noises. Electromechanical relay equipment was located in an adjacent room and controlled stimulus events and response recording.

\section{Procedure}

Throughout the experiment, all sessions lasted $50 \mathrm{~min}$. The response lever was removed from the chambers during preliminary training. Sessions 1-3 consisted of dipper training, in which the dipper containing .1 cc of $10 \%$ sucrose solution (by weight) was available on a variable-time 1-min schedule. Sessions 4-7 consisted of food-cup training, in which a 45-mg food pellet was delivered into the food cup on a variable-time 1-min schedule. The response lever was then introduced and remained in the chambers for the rest of the experiment. Subjects were conditioned to barpress for 6-sec access to the sucrose solution. Barpressing was reinforced continuously during Sessions 8-12, on a variable-interval 30 -sec schedule during Sessions 9-11, and on a variable-interval 2-min schedule during Sessions 12-17.

For the testing phase of the experiment the 24 rats were randomly assigned to two groups of 12 subjects each. In each of the 24 testing sessions, subjects in both groups were exposed to eight presentations of an $8-\mathrm{sec}$ light CS while responding on the variable-interval 2-min schedule of reinforcement. The mean interval between CS onsets was $6.25 \mathrm{~min}$. Each CS terminated with the delivery of a food pellet US for the $100 \%$ CS-US group. For the $50 \%$ CS-US group, four of the eight CS presentations in each session terminated with a food pellet US. The order of CS-US pairings and CS-alone trials was changed every session for the $50 \%$ CS-US group. Presentations of the CS and US did not affect the variable-interval 2-min schedule of sucrose reinforcement for either group.

Following each of the 24 sessions of the testing phase, the total number of responses during the eight $8-\mathrm{sec}$ CS intervals and the total number of responses during the eight 8 -sec intervals immediately preceding the CS presentations (pre-CS intervals) were recorded for each subject. From these totals, response rates during the pre-CS and CS intervals were computed for each subject. Inflection ratios were also calculated for each subject by dividing the CS rate by the sum of the CS rate and the pre-CS rate. These inflection ratios provided a measure of change in the response rates during the CS while controlling for pre-CS rates. An inflection ratio less than .50 meant that the subject was responding at a lower rate during the CS than during the immediately preceding interval, and was considered evidence of positive conditioned suppression.

\section{RESULTS}

Figure 1 shows the mean response rates during the pre-CS and CS intervals for each group over blocks of four sessions. An analysis of variance was performed on these response rates over the six blocks of sessions. This analysis revealed a significant main effect of interval $(\mathrm{F}=7.35, \mathrm{df}=1 / 22, \mathrm{p}<.05)$ and a significant Groups by Interval interaction $(F=7.05, \mathrm{df}=1 / 22, \mathrm{p}<.05)$. Tests of simple main effects revealed no difference in pre-CS rates between groups, no difference in CS rates between groups, no difference between the pre-CS and CS response rates for the $50 \%$ CS-US group, and significantly lower response rates during the CS intervals than during the pre-CS intervals for the $100 \%$ CS-US group.

Group mean inflection ratios over blocks of four sessions are shown in Figure 2. As can be seen in Figure 2, the mean inflection ratios of the $100 \%$ CS-US group indicate substantial suppression of response rates during the CS, while mean inflection ratios of the $50 \%$ CS-US group indicate, at most, a slight suppression of response rates during the CS. An analysis of variance on inflection ratios over the six blocks of sessions confirmed the statistical reliability of the differences between group mean inflection ratios $(F=6.73, \mathrm{df}=1 / 22$, $\mathrm{p}<.05)$. A significant main effect of blocks $(F=4.01$, $\mathrm{df}=5 / 110, \mathrm{p}<.01)$ reflected the decline in mean inflection ratios across groups. The analysis did not reveal a significant interaction of Groups by Blocks.

The analysis of variance of inflection ratios revealed more response suppression during the CS for the $100 \%$ CS-US group than for the 50\% CS-US group. In order to

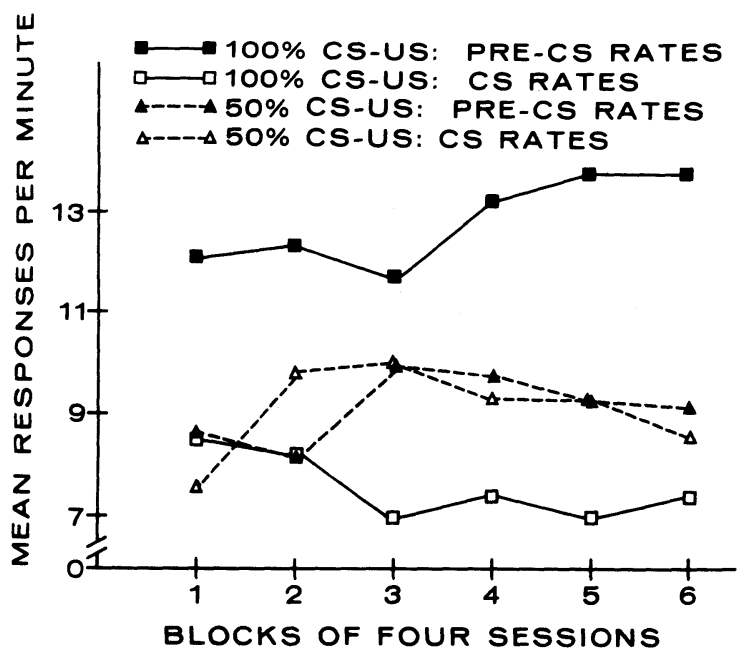

Figure 1. Mean response rates for $100 \%$ and $50 \%$ CS-US groups are shown over blocks of four sessions. Mean response rates during the pre-CS and CS intervals are plotted separately for each group. 


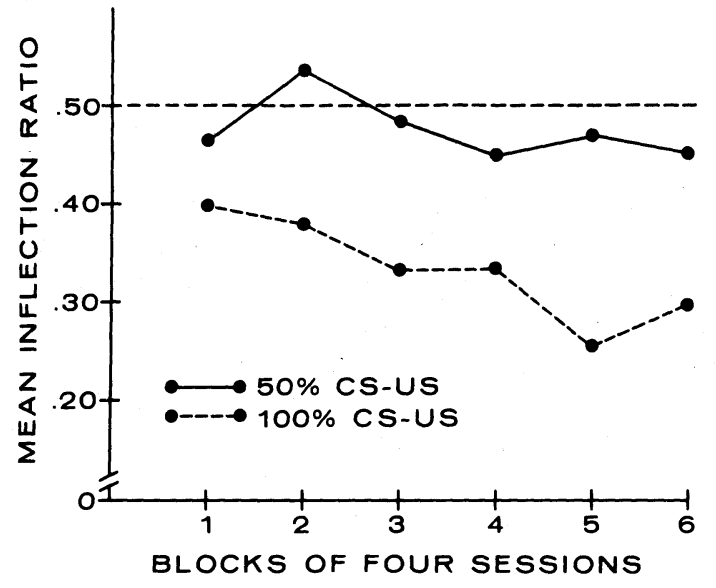

Figure 2. Mean inflection ratios for $100 \%$ and $50 \%$ CS-US groups are shown over blocks of four sessions.

determine if each group's inflection ratios represented significant response suppression, one-tailed $t$ tests were performed on the difference between each group's mean inflection ratio for the last block and .50. These $t$ tests revealed that the $100 \%$ CS-US group had inflection ratios significantly below .50 $(\mathrm{t}=3.91, \mathrm{p}<.01)$ but the $50 \%$ CS-US group's inflection ratios did not significantly differ from $.50(\mathrm{t}=1.09, \mathrm{p}<.20)$.

\section{DISCUSSION}

The analyses of response rates and inflection ratios yielded essentially the same information. Positive conditioned suppression was observed with $100 \%$ pairings of a short CS and a positive US, but not with $50 \%$ CS-US pairings. In light of the results of the present experiment, the enhancement observed in the $33 \%$ and $66 \%$ groups of Meltzer and Hamm (1974) following a shift in CS duration from $2 \mathrm{~min}$ to $8 \mathrm{sec}$ seems most likely to be due to the previous experience with the 2 -min CS.

Two aspects of the present results differ from those of negative conditioned suppression studies. First, the present experiment found an effect of percentage of CS-US pairings between groups of subjects, while between-subject effects have not been found when shock is used as the US. Second, the $50 \%$ CS-US group failed to develop positive conditioned suppression. In studies of negative conditioned suppression, $50 \%$ pairings of the CS and shock have been sufficient to develop considerable suppression (Wagner, Siegel, \& Fein, 1967).

One factor which may contribute to these differences between positive and negative conditioned suppression is the intensity of the US. It is difficult to compare the intensity of a positive US, such as food, to the intensity of a negative US, such as shock. Thus, the importance of this factor for the dif- ferences between positive and negative conditioned suppression is difficult to assess.

A second possible explanation for the differences between the present experiment and those manipulating the percentage of pairings of a CS and a negative US is suggested by the analysis of Meltzer and Hamm (1974). According to their analysis, the 8-sec CS elicited a response-facilitating emotional response and a second response-interfering response. In the $100 \%$ CS-US group, these competing response tendencies combined to produce a net effect of positive conditioned suppression. Reducing the percentage of CS-US pairings, as in the 50\% CS-US group, led to a net effect of neither conditioned suppression nor conditioned enhancement. A reduction in the percentage of pairings may weaken the strength of the interfering response, or may weaken the strength of both the interfering response and the facilitating emotional response.

\section{REFERENCES}

Azrin, N. H., \& Hake, D. F. Positive conditioned suppression: Conditioned suppression using positive reinforcers as the unconditioned stimuli. Journal of the Experimental Analysis of Behavior, 1969, 12, 167-173.

Brimer, C. J., \& Dockrill, F. J. Partial reinforcement and the CER. Psychonomic Science, 1966, 5, 185-186.

Estes, W. K., \& SkInner, B. F. Some qualitative properties of anxiety. Journal of Experimental Psychology, 1941, 29. $390-400$.

GELlER, I. Conditioned suppression in goldfish as a function of shock-reinforcement schedule. Journal of the Experimental Analysis of Behavior, 1964, 7, 345-349.

HeARST, E., \& Jenkins, H. M. Sign-tracking: The stimulusreinforcer relation and directed action. Austin, Tex: Psychonomic Society, 1974.

Huton, A. Partial reinforcement of a conditioned emotional response in rats. Journal of Comparative and Physiological Psychology, 1969, 69, 253-260.

Meltzer, D., \& Brahlek, J. A. Conditioned suppression and conditioned enhancement with the same positive UCS: An effect of CS duration. Journal of the Experimental Analysis of Behavior, 1970, 13, 67-74.

Meltzer, D., \& HaMm, R. J. Conditioned enhancement as a function of the percentage of CS-US pairings and CS duration. Bulletin of the Psychonomic Society, 1974, 4, 467-470.

Wagner, A. R., Siegel, L. S., \& Fein, G. G. Extinction of conditioned fear as a function of the percentage of reinforcement. Journal of Comparative and Physiological Psychology, 1967, 63, 160-164.

WILlis, R. D. The partial reinforcement of conditioned suppression. Journal of Comparative and Physiological Psychology, 1969, 68, 289-295.

Willis, R. D., \& Lundin, R. W. Conditioned suppression in the rat as a function of shock reinforcement schedule. Psychonomic Science, 1966, 6, 107-108.

(Received for publication March 31, 1977.) 